\title{
Subspecialty surveillance of long-term course of small and moderate muscular ventricular septal defect: heterogenous practices, low yield
}

\author{
Erik L Frandsen, Aswathy V House, Yunbin Xiao, David A Danford and Shelby Kutty*
}

\begin{abstract}
Background: No expert consensus guides practice for intensity of ongoing pediatric cardiology surveillance of hemodynamically insignificant small and moderate muscular ventricular septal defect (mVSD). Therefore, despite the well-established benign natural history of mVSD, there is potential for widely divergent follow up practices. The purpose of this investigation was to evaluate (1) variations in follow up of mVSD within an academic children's hospital based pediatric cardiology practice, and (2) the frequency of active medical or surgical management resulting from follow up of mVSD.

Methods: We retrospectively reviewed records of 600 patients with isolated mVSD echocardiographically diagnosed between 2006 and 2012. Large mVSD were excluded $(n=4)$. Patient age, gender, echocardiographic findings, provider, recommendations for follow up, and medical and surgical management were tabulated at initial and follow up visits. Independent associations with follow up recommendations were sought using multivariate analysis.
\end{abstract}

Results: Initial echocardiography showed small single mVSD in 509 (85\%), multiple small mVSD in 60 (10\%), and small-to-moderate or moderate single mVSD in 31 (5\%). The mean age at diagnosis was 15.9 months (0-18.5 years) and 25.7 months ( $0-18.5$ years) at last follow up. There was slight female predominance (56.3\%). Fourteen pediatric cardiology providers recommended 316 follow up visits, 259 of which were actually accomplished. There were 37 other unplanned follow up visits. No medical or surgical management changes were associated with any of the follow up visits. The proportion of patients for whom follow up was advised varied among providers from 11 to $100 \%$. Independent associations with recommendation for follow up were limited to the identity and clinical volume of the provider, age of the patient, and the presence of multiple, small-to-moderate, or moderate mVSD.

Conclusions: In this large series of moderate or smaller mVSD, pediatric cardiology follow up was commonly recommended but resulted in no active medical or surgical management. Major provider based inconsistency in intensity of follow up of mVSD was identified, but is difficult to justify.

Keywords: Congenital heart disease, Muscular ventricular septal defect, Echocardiography, Follow-up practices

\section{Background}

Ventricular septal defect (VSD) is the most common isolated congenital cardiac defect, representing up to $40 \%$ of congenital cardiac defects diagnosed in infancy [1]. Defects located within the muscular septum constitute the more frequently seen type of VSD [2]. The natural

\footnotetext{
* Correspondence: skutty@unmc.edu

Division of Pediatric Cardiology, University of Nebraska College of Medicine and Children's Hospital and Medical Center, 8200 Dodge St, Omaha, NE
} 68114 , USA

history of muscular ventricular septal defects (mVSD) is well described. Up to $76 \%$ undergo spontaneous closure by the end of the first year of life [3-7], a large proportion of which close by six months of age $[3,4,8]$. The rates of spontaneous closure are higher for mVSD compared to membranous VSD [6,7]. In a series of apical mVSD's diagnosed between 1 day and 13 years of age, up to $44 \%$ spontaneous closure rate was reported within 3 years of diagnosis [4]. A 1.8\% risk of infective endocarditis has been reported for VSD, mostly occurring in the 
perimembranous type [9]. Neumayer et al. reported no endocarditis complication for isolated mVSDs in their series [10]. The majority of small mVSD do not require surgical management, and for those small defects that remain patent, long-term complication rates are minimal [9-12].

Despite the well-described natural history and benign course, no expert consensus guides practice for intensity of ongoing pediatric cardiology surveillance of small mVSD. A survey by Smith and Qureshi demonstrates the general divergence of opinion regarding follow up for congenital heart defects [13]. Follow up practice patterns of hemodynamically insignificant mVSD have not been specifically studied previously. The purpose of this investigation is to evaluate (1) variations in follow up of mVSD within an academic children's hospital based group pediatric cardiology practice, and (2) the frequency of active medical or surgical management resulting from follow up of mVSD.

\section{Methods}

This was a retrospective cohort study performed in a university affiliated academic children's hospital, in accordance with the ethical standards laid down in the 1964 Declaration of Helsinki and its later amendments. The Institutional Review Board of the University of Nebraska Medical Center approved the study. Informed consent was waived for subjects included. Pediatric cardiology databases were used to identify patients who had echocardiographic diagnosis of mVSD between 2006 and 2012.

In accordance with the recommended standards and guidelines for pediatric echocardiography set by the Intersocietal Accreditation Commission for Echocardiography Laboratories (ICAEL) and the American Society of Echocardiography, echocardiography was performed by registered cardiac sonographers, and reported by board certified pediatric cardiologists. Only those patients who had hemodynamically insignificant mVSD on echocardiography, evidenced by restrictive left to right shunting, absence of ventricular hypertrophy, and normal pulmonary artery pressure were included. Patients with age appropriate patent foramen ovale and patent ductus arteriosus were also included. Specific exclusion criteria consisted of (1) patients with additional VSD located in areas besides the muscular septum, (2) patients with any associated cardiac lesion, (3) patients with large mVSD, and 4) patients with previous cardiothoracic surgery.

Patient age, gender, echocardiographic findings, PC provider, recommendations for follow up, and medical and surgical management at initial and follow up visits were obtained from medical records review. Patients were categorized based on echocardiographic reported size of mVSD (small, small-to-moderate, moderate, large) and number of mVSD (isolated or multiple) at initial diagnosis. Patient age was categorized as younger ( $<3$ months at entry into study) vs. older. Pediatric cardiology providers were categorized based on training (physician pediatric cardiologist vs. physician assistant), on clinical volume during the study ( $\geq 50$ cases vs. $<50$ cases), and on echocardiographic expertise (official interpreter of echocardiograms vs. non-interpreter).

\section{Follow-up}

At initial visit and each subsequent follow up, pediatric cardiology provider visit records were reviewed. The following information was obtained from the records for each provider: (1) continued presence or spontaneous closure of mVSD by clinical exam and by echocardiography, (2) if the mVSD remained open, provider impression regarding hemodynamic effects of the defect (significant or insignificant), (3) recommendation for follow up, (4) time until next recommended follow up, and (5) recommendation for medical or surgical management.

\section{Statistics}

Descriptive statistics for categorical variables are reported as frequency and percentage. Univariate comparisons of outcome were made among dichotomous variables using the Chi square test. Candidate independent variables were selected based on univariate correlation $(\mathrm{p}<0.15)$, and were incorporated into a multivariate logistic regression to generate a model associating them with follow up recommendation. Alpha to enter and alpha to exclude variables from the stepwise process were both 0.15 . A p value of $<0.05$ represented significance. Statistical analysis was performed with commercially available computer software (Minitab 16.0, Minitab Inc., State College, PA).

\section{Results}

\section{Patient characteristics}

After exclusion of 4 patients with large mVSD, the study population consisted of 600 patients with mVSD. None of the patients had a swiss cheese septum. There were 262 males (43.7\%) and 338 females (56.3\%). The mean age at diagnosis was 15.9 months (0-18.5 years) and at last follow up 25.7 months (0-18.5 years). Initial Echo showed isolated small mVSD in 509 patients $(85 \%)$, multiple small mVSD in 60 (10\%), isolated small-tomoderate $\mathrm{mVSD}$ in $12(2 \%)$, and isolated moderate mVSD in 19 (3\%) patients (Table 1).

\section{Follow-up patterns}

In all, 316 follow up visits were recommended by fourteen pediatric cardiology providers, of which 259 were actually accomplished. There were 37 other unplanned 
Table 1 Characteristics of muscular VSD and associated rate of follow up recommendation

\begin{tabular}{lll}
\hline mVSD size & $\begin{array}{l}\text { Number of } \\
\text { patients }\end{array}$ & $\begin{array}{l}\text { Rate of follow up } \\
\text { recommendation (\%) }\end{array}$ \\
\hline Isolated small & $509(85 \%)$ & 32 \\
Isolated small-to-moderate & $12(2 \%)$ & 42 \\
Isolated moderate & $19(3 \%)$ & 84 \\
Multiple small & $60(10 \%)$ & 58 \\
\hline
\end{tabular}

mVSD muscular ventricular septal defect.

follow up visits. No recommendations for medical or surgical management were made at any of the follow up visits. The mean follow up duration was 1.4 years (range 1 month to 5 years). For those patients who were dismissed from follow up, the mean interval from initial visit to dismissal was 0.82 years (range 0 days to 12.3 years). There were $12 \mathrm{MD}$ (evaluated $85 \%$ of small mVSDs) and 2 non-MD physician assistant providers (evaluated $15 \%$ of small mVSDs).

Follow up was recommended for patients with an isolated small mVSD at a rate of $32 \%$, versus $68 \%$ for small-to-moderate and moderate isolated mVSD, and $58 \%$ for multiple small mVSD ( $<<0.001$, Table 1$)$. Younger age at first visit was associated with greater rate of recommendation for follow up. The mean age at first visit for patients who were recommended follow up was 11.4 months vs. 18.4 months for patients who were not recommended follow up $(\mathrm{p}=0.006)$.

There was significant variability in follow up recommendations between providers, ranging from 11-100\% (Table 2). Independent associations with recommendation for follow up were limited to high clinical volume provider, the mVSD characteristics (multiple mVSD, isolated small-to-moderate, or isolated moderate mVSD), and patient age under 3 months at first visit (Table 3). Patient gender, echo-reading provider, and non-MD provider did not appear to be independently related to subspecialty follow-up recommendations (Table 3 ).

\section{Discussion}

No clear consensus exists about the value of follow up for small mVSD [14]. Of 52 respondents to a small survey conducted among pediatric cardiology providers in the UK, the majority would follow up a hemodynamically insignificant VSD in three years, $15 \%$ would do so in one year, and less than $10 \%$ would dismiss the patient upon diagnosis [13]. Our experience confirmed the striking variability in actual practice that the survey would lead us to expect. In this report, individual providers recommended pediatric cardiology follow up for as few as $11 \%$ and as many as $100 \%$ of their patients.

Small mVSD are common. Studies prior to the routine use of echocardiography determined an incidence of 1.33.3 VSD per 1000 live births $[15,16]$, but with the advent of echocardiography, incidence has increased significantly $[8,17,18]$. Because the population of children with small mVSD is large, a policy of routine pediatric cardiology follow up would consume substantial resources in the subspecialty outpatient clinic. The benefits of this investment are not obvious; given the natural history studies that show uncomplicated small mVSD carries a high likelihood of spontaneous closure [3-7,11,12], and little if any prospect for clinical deterioration $[11,12]$. It is impressive that the provider with the largest practice had

Table 2 Patient characteristics and pediatric cardiology provider follow up practice patterns for small muscular VSD

\begin{tabular}{llllll}
\hline $\begin{array}{l}\text { Pediatric cardiology } \\
\text { provider }\end{array}$ & $\begin{array}{l}\text { Number of patients } \\
\text { for each provider }\end{array}$ & $\begin{array}{l}\text { Number of patients } \\
\text { with small mVSD }\end{array}$ & $\begin{array}{l}\text { Percentage of patients } \\
\text { with small mVSD }\end{array}$ & $\begin{array}{l}\text { Number of follow up } \\
\text { recommendations }\end{array}$ & $\begin{array}{l}\text { Percentage of patients } \\
\text { for which follow up was } \\
\text { recommended }\end{array}$ \\
\hline 1 & 4 & 4 & 100 & 4 & 100 \\
2 & 274 & 244 & 99 & 31 & 11 \\
3 & 22 & 82 & 24 & 89 \\
4 & 27 & 72 & 87 & 27 & 33 \\
5 & 83 & 5 & 83 & 2 & 33 \\
6 & 6 & 69 & 79 & 59 & 68 \\
7 & 87 & 28 & 74 & 16 & 42 \\
8 & 38 & 4 & 80 & 3 & 60 \\
9 & 5 & 8 & 73 & 8 & 73 \\
10 & 11 & 11 & 85 & 3 & 23 \\
11 & 13 & 18 & 78 & 21 & 91 \\
12 & 23 & 13 & 72 & 17 & 94 \\
14 & 18 & 6 & 100 & 4 & 67 \\
\hline
\end{tabular}

mVSD muscular ventricular septal defect. 
Table 3 Independent associations with recommendation for follow up

\begin{tabular}{|c|c|c|c|c|c|}
\hline \multirow[b]{2}{*}{ Variable } & \multicolumn{3}{|c|}{ Univariate analysis } & \multicolumn{2}{|c|}{ Multivariate analysis } \\
\hline & $\mathrm{N}_{\text {with }}$ (\% Follow-up recommended) & $\mathrm{N}_{\text {without }}$ (\% Follow-up recommended) & $\mathbf{p}$ & Odds ratio $(95 \% \mathrm{CL})$ & $p$ \\
\hline High volume provider & $444(26.3)$ & $156(66.0)$ & $<0.001$ & $5.23(3.44-7.93)$ & $<0.001$ \\
\hline Non MD provider & $88(31.8)$ & $512(37.5)$ & 0.303 & -—-ー- & -—— \\
\hline Echo reader provider & $451(33.7)$ & $149(45.6)$ & 0.009 & $1.20(0.78-1.84)$ & 0.409 \\
\hline Male gender & $262(36.3)$ & $338(37.0)$ & 0.855 & -—-—- & -—— \\
\hline Multiple mVSD & $60(58.3)$ & $540(34.3)$ & $<0.001$ & $0.38(0.21-0.68)$ & 0.001 \\
\hline $\begin{array}{l}\text { Small-moderate or } \\
\text { moderate mVSD }\end{array}$ & $31(67.7)$ & $569(35.0)$ & $<0.001$ & $0.27(0.12-0.63)$ & 0.002 \\
\hline Age $<3$ months & $304(44.1)$ & $296(29.0)$ & $<0.001$ & $0.57(0.39-0.83)$ & 0.003 \\
\hline
\end{tabular}

the lowest follow up rate of $11 \%$, while providers with the smaller clinical practice had the higher rates. This would suggest that provider experience might be associated with better resource utilization, perhaps driven by greater confidence.

Our data confirms that among a large number of pediatric cardiology follow up visits for mVSD, we could identify no case in which it resulted in active medical or surgical management of the condition. Certain clinical features, such as younger age of the patient, number of defects, and any suggestion that the defect may be moderate size, appeared to influence the likelihood that follow up would be arranged without identifying a subgroup in which active medical or surgical management actually took place. Patients with multiple small mVSD in this series were recommended follow up more often than isolated small mVSD. Patients with isolated moderate sized mVSD were recommended follow up at a greater rate than those with isolated small-to-moderate and small sized defects. We speculate that perceived differences in spontaneous closure rates (between defects of different sizes and numbers) might have influenced follow up recommendations. It is known that age at diagnosis and method of diagnosis influence reported spontaneous closure rates for VSD $[3,6,7,19,20]$. Young age at diagnosis was a significant predictor of spontaneous closure [19,21].

Even after accounting for age of the patient and perceived size and number of muscular VSD's, we found that follow-up recommendations are highly provider dependent. We interpret this as a sign that follow up recommendations for $\mathrm{mVSD}$ in our series were not evidence-based. Recently, the implementation of standardized clinical assessment management plans (SCAMPS) methodology reduced resource use and practice variation in the outpatient evaluation of pediatric cardiology chest pain [22]. A similar process could be applied to establish care standards for subspecialty surveillance of small mVSD.

Living in a society that presumes that more healthcare is better healthcare, and supposing that any cardiac defect represents a threat, concerned parents may exert pressure on pediatric cardiologists to provide ongoing subspecialty care for small mVSD. No physical harm likely arises from asking these patients to return for subspecialty follow-up, but because there were no interventions for those in this series who did return, it is intriguing to speculate whether harm of other sorts might be done. Pediatric cardiologists have long been sensitive to concerns that if benign conditions are permitted to be understood as threats, that inappropriate restrictions might be imposed on the patients by wellmeaning parents, other care providers, or school personnel [23]. It is beyond the scope of this investigation to decide if diligent subspecialty follow-up for small mVSD might foster consequences akin to 'cardiac nondisease' described years ago for innocent murmur $[23,24]$. High clinical volume providers in this series may have already concluded based on experience that for the vast majority of small mVSD, the costs and potential for adverse psychological impact associated with routine subspecialty surveillance outweigh any benefits.

\section{Limitations}

This study has the limitations associated with a retrospective cohort study. Several other factors may have impacted follow up patterns on a case-by-case basis. This type of investigation does not account for everything that plays into pediatric cardiology provider decision to recommend follow up. Neither does it provide timelines for follow up of small hemodynamically insignificant mVSDs. Clinical scenarios for each patient may be different, which influences pediatric cardiology provider decisions.

\section{Conclusion}

Wide practice variation was observed in the surveillance frequency for small and moderate sized mVSD within a 14-provider pediatric cardiology group, in the absence of active medical or surgical management. Allowances 
should always be made permitting practice variations in exceptional cases, so an occasional mVSD will receive follow up, but this investigation does not support a practice of regularly scheduled subspecialty surveillance.

\section{Abbreviation}

mVSD: muscular ventricular septal defect.

\section{Competing interests}

The authors declare that they have no competing interests.

\section{Authors' contributions}

$\mathrm{EF}, \mathrm{DD}$, and SK designed the study, EF, AVH and YX performed data collection and analysis, EF and SK drafted the manuscript and did critical revisions, all authors read and approved the final manuscript.

\section{Acknowledgment}

The authors appreciate the assistance of Jesse Fisher. SK receives support from the American College of Cardiology Foundation, the Children's Hospital and Medical Center Foundation, and the American Heart Association.

Received: 21 May 2014 Accepted: 25 September 2014

Published: 4 November 2014

\section{References}

1. Hoffman JI: Incidence of congenital heart disease: I. Postnatal incidence. Pediatr Cardiol 1995, 16:103-113.

2. Shirali GS, Smith EO, Geva T: Quantitation of echocardiographic predictors of outcome in infants with isolated ventricular septal defect. Am Heart J 1995, 130:1228-1235.

3. Hiraishi S, Agata Y, Nowatari M, Oguchi K, Misawa H, Hirota H, Fujino N, Horiguchi Y, Yashiro K, Nakae S: Incidence and natural course of trabecular ventricular septal defect: two-dimensional echocardiography and color doppler flow imaging study. J Pediatr 1992, 120:409-415.

4. Atalay S, Imamoglu A, Dilek L, Altug N, Tutar E, Gumus H: Congenital isolated apical ventricular septal defects. Angiology 1998, 49:355-359.

5. Axt-Fliedner R, Schwarze A, Smrcek J, Germer U, Krapp M, Gembruch U: Isolated ventricular septal defects detected by color doppler imaging Evolution during fetal and first year of postnatal life. Ultrasound Obstet Gynecol 2006, 27:266-273.

6. Mehta AV, Chidambaram B: Ventricular septal defect in the first year of life. Am J Cardio/ 1992, 70:364-366.

7. Moe DG, Guntheroth WG: Spontaneous closure of uncomplicated ventricular septal defect. Am J Cardiol 1987, 60:674-678.

8. Ekici F, Tutar E, Atalay S, Arsan S, Ozcelik N: The incidence and follow-up of isolated ventricular septal defect in newborns by echocardiographic screening. Turk J Pediatr 2008, 50:223-227.

9. Gabriel HM, Heger M, Innerhofer P, Zehetgruber M, Mundigler G, Wimmer M, Maurer G, Baumgartner $\mathrm{H}$ : Long-term outcome of patients with ventricular septal defect considered not to require surgical closure during childhood. J Am Coll Cardiol 2002, 39:1066-1071.

10. Neumayer U, Stone S, Somerville J: Small ventricular septal defects in adults. Eur Heart J 1998, 19:1573-1582.

11. Kidd L, Driscoll DJ, Gersony WM, Hayes CJ, Keane JF, O'Fallon WM, Pieroni DR, Wolfe RR, Weidman WH: Second natural history study of congenital heart defects. Results of treatment of patients with ventricular septal defects. Circulation 1993, 87:138-151.

12. Onat $T$, Ahunbay G, Batmaz G, Celebi A: The natural course of isolated ventricular septal defect during adolescence. Pediatr Cardiol 1998, 19:230-234

13. Smith BG, Qureshi SA: Paediatric follow-up of haemodynamically insignificant congenital cardiac lesions. J Paediatr Child Health 2012, 48:1082-1085

14. Cantinotti M, Assanta N, Murzi B, Lopez L: Controversies in the definition and management of insignificant left-to-right shunts. Heart 2014 100:200-205.

15. Hoffman Jl, Rudolph AM: The natural history of ventricular septal defects in infancy. Am J Cardiol 1965, 16:634-653.
16. Kerrebijn KF: Incidence in infants and mortality from congenital malformations of the circulatory system. Acta Paediatr Scand 1966, 55:316-320

17. Meberg A, Otterstad JE, Froland G, Sorland S, Nitter-Hauge S: Increasing incidence of ventricular septal defects caused by improved detection rate. Acta Paediatr 1994, 83:653-657.

18. Zhao QM, Ma XJ, Jia B, Huang GY: Prevalence of congenital heart disease at live birth: an accurate assessment by echocardiographic screening. Acta Paediatr 2013, 102:397-402.

19. Atalay S, Tutar E, Ekici F, Nacar N: Spontaneous closure of small apical muscular ventricular septal defects. Turk J Pediatr 2005, 47:247-250

20. Atik E: Small ventricular septal defect. long-term expectant clinical management. Ara Bras Cardiol 2009, 92:396-399. 413-396, 429-332.

21. Turner SW, Hornung T, Hunter S: Closure of ventricular septal defects: a study of factors influencing spontaneous and surgical closure. Cardiol Young 2002, 12:357-363.

22. Friedman KG, Kane DA, Rathod RH, Renaud A, Farias M, Geggel R, Fulton DR, Lock JE, Saleeb SF: Management of pediatric chest pain using a standardized assessment and management plan. Pediatrics 2011, 128:239-245.

23. Bergman AB, Stamm SJ: The morbidity of cardiac nondisease in schoolchildren. N Engl J Med 1967, 276:1008-1013.

24. Geggel RL, Horowitz LM, Brown EA, Parsons M, Wang PS, Fulton DR: Parental anxiety associated with referral of a child to a pediatric cardiologist for evaluation of a still's murmur. J Pediatr 2002, 140:747-752.

doi:10.1186/1471-2431-14-282

Cite this article as: Frandsen et al:: Subspecialty surveillance of longterm course of small and moderate muscular ventricular septal defect: heterogenous practices, low yield. BMC Pediatrics 2014 14:282.

\section{Submit your next manuscript to BioMed Central and take full advantage of:}

- Convenient online submission

- Thorough peer review

- No space constraints or color figure charges

- Immediate publication on acceptance

- Inclusion in PubMed, CAS, Scopus and Google Scholar

- Research which is freely available for redistribution 